\title{
Role of local structure on motions on the potential energy landscape for a model supercooled polymer
}

\author{
Tushar S. Jain and Juan J. de Pablo ${ }^{\text {a) }}$ \\ Department of Chemical and Biological Engineering, University of Wisconsin-Madison, Madison, \\ Wisconsin 53706
}

(Received 27 August 2004; accepted 16 February 2005; published online 5 May 2005)

\begin{abstract}
We have conducted detailed Monte Carlo and molecular dynamics simulations of a model glass forming polymeric system near its apparent glass transition temperature. We have characterized the local structure of the glass using a Voronoi-Delaunay analysis of local particle arrangements. After a perturbative face elimination, we find that a significant fraction of Voronoi polyhedra consist of 12 pentagonal faces, a sign of icosahedral ordering. Further, we have identified metabasins of particle vibrations on the potential energy landscape on the basis of persistence of particle positions and neighbors over a simulated trajectory. We find that the residence times for vibrations are correlated with a particular Voronoi volume and number of neighbors of a particle; the largest metabasins correspond to particles whose average Voronoi volume is close to the value expected on the basis of the density, and whose approximate number of neighbors is close to 12 . The local distortion around a particle, measured in terms of the tetrahedricity of the Delaunay simplices, reveals that the particles with a higher degree of local distortion are likely to transition faster to a neighboring metabasin. In addition to the transition between metabasins, we have also examined the influence of vibrations at inherent structures (IS) on the local structure, and find that the the frequency modes at the IS exhibit the greatest curvature with respect to the local structure. We believe that these results establish an important connection between the local structure of glass formers and the activated dynamics, thereby providing insights into the origins of dynamic heterogeneities. (C) 2005 American Institute of Physics. [DOI: 10.1063/1.1888505]
\end{abstract}

\section{INTRODUCTION}

One of the long-standing goals of theoretical and experimental research on glasses has been to establish a relation between local structure and the observed dynamics. The relation between a particle's mobility and its free volume is a central tenet of the free-volume theory. ${ }^{1-3}$ Experiments ${ }^{4}$ and computer simulations ${ }^{5}$ of glass formers indicate that the $a v$ erage structure exhibits no dramatic changes as the system goes through the glass transition even though the dynamics become increasingly heterogeneous, ${ }^{6,7}$ thereby indicating an apparent lack of dependence of the dynamics on the structure. Since molecular simulations have access to detailed particle trajectories and positions, they are well-suited for the investigation of local particle properties, structural arrangements, and dynamic heterogeneities. A detailed analysis of the dynamics has clearly revealed the presence of dynamic heterogeneities for several glass formers. ${ }^{8-11}$ Recent studies ${ }^{12,13}$ have attempted to describe the relaxation of glasses using the concept of "dynamic facilitation" whereby mobile excitations in one region confer mobility on the surrounding regions leading to the presence of dynamic heterogeneities. The fundamental origin of the mobile excitations, however, is still an open fundamental question. ${ }^{13}$ Several molecular simulations have also examined the change of local structure and free volume of glass forming liquids as the

\footnotetext{
${ }^{a)}$ Author to whom correspondence should be addressed. Electronic mail: tsjain@cae.wisc.edu
}

glass transition temperature $\left(T_{g}\right)$ is approached. In these simulations, particles have been classified into liquid- or solid-like categories on the basis of free volume, and various measures of shape ${ }^{14}$ and distortion ${ }^{15}$ of the Voronoi polyhedra and Delaunay simplices. ${ }^{16,17}$ Structural signatures in the form of percolation thresholds ${ }^{14,18,19}$ of Delaunay networks and increase in icosahedral ordering ${ }^{20-22}$ have been observed near $T_{g}$. Further, a higher degree of icosahedral order has been observed when the cooling rate is reduced. ${ }^{23}$ Experimentally, the degree of nonexponential relaxation and fragility have been found to exhibit a correlation with chemical structure for a number of glass formers, ${ }^{24-27}$ with a general indication that structures presenting a greater steric hindrance exhibit a greater degree of nonexponentiality.

Another useful theoretical framework for the study of glasses is the potential energy landscape (PEL) ${ }^{28,29}$ In this framework, the PEL is partitioned into energy basins connected by saddle points that lead the system from one basin to another. One view of the dynamics in glasses assumes a separation of time scales as the system approaches the glass transition temperature, such that motion at short times occurs via intrabasin vibrations about a particular inherent structure (local potential energy minimum), and long-time motion occurs via infrequent activated jumps over saddle points into neighboring basins. An elaboration of this concept has led to the picture of "metabasins," of several local minima separated by low energy barriers, and the $\alpha$ relaxation occurs via jumps between neighboring 
metabasins. Past studies have demonstrated the influence of the PEL on the dynamics of glass formers, with the appearance of a dynamical regime exhibiting a separation of intrabasin and interbasin motion below a certain crossover temperature. ${ }^{31,32}$ Transitions between adjacent minima have also been shown to exhibit cooperative motion. ${ }^{33,34}$ More recent studies have established a link between the topography of the PEL and the dynamics for a binary Lennard-Jones glass. ${ }^{30}$ Investigation of the energy barriers on the PEL for a model bulk and free-standing film polymer glass have revealed significant differences arising due to confinement by free interfaces. ${ }^{34}$

However, possible links between the local structure and activated dynamics on the PEL have not been extensively explored, though earlier studies have indicated the influence of potential energy, ${ }^{35}$ icosahedral ordering, ${ }^{36}$ and $\mathrm{H}$ bonding ${ }^{37,38}$ on mobility. This work presents results for a model glass forming polymer that establish a relation between the periods of vibration on the PEL and the local structure of the particles in the inherent structures (IS). These results indicate that the time of vibration of a particle in a so-called "metabasin" is correlated with the structure of its Voronoi polyhedron and the distortion of the Delaunay simplices to which it belongs. It is shown that the longest metabasins occur for particles that have approximately 12 geometrical neighbors and a Voronoi volume that is close to the value expected from the system's density.

The paper is organized as follows. In Sec. II, we describe the model employed in this study, and discuss some of the canonical "glassy" features that are exhibited by the model. Section III discusses the local structure of the system in terms of the Voronoi-Delaunay analysis. In Sec. IV, we present the algorithm for calculating the particle metabasins of vibration on the PEL, and discuss the correlations between the structure of the Voronoi-Delaunay constructions and the lengths of the metabasins. Section V discusses the influence of the normal modes of vibration at the inherent structures on the system structure. Finally, we conclude in Sec. VI and discuss some of the implications of this study.

\section{MODEL AND SIMULATION DETAILS}

The model employed in this work consists of polymer chains of spherical interaction sites of diameter $\sigma$. Nonbonded sites interact through a modified ${ }^{39}$ 6-12 LennardJones potential with characteristic energy $\epsilon$, and bonded sites interact through a harmonic potential,

$$
\begin{aligned}
& U_{i j}^{\mathrm{nb}}=4 \varepsilon\left[\left(\frac{\sigma}{r_{i j}}\right)^{12}-\left(\frac{\sigma}{r_{i j}}\right)^{6}\right]+c_{0} \varepsilon+c_{1} \varepsilon\left(2.5-\frac{\sigma}{r_{i j}}\right), \\
& U_{i j}^{\mathrm{b}}=k_{h}\left(r_{i j}-l_{0}\right)^{2},
\end{aligned}
$$

where $c_{1}=0.016316891, c_{2}=0.038999477, k_{h}=1000 \varepsilon / \sigma^{2}$ is the spring constant, the equilibrium bond length is $l_{0}=\sigma$, and $r_{i j}$ is the distance between the interaction sites. The cutoff distance for the Lennard-Jones potential is $2.5 \sigma$. The modified potential is required in order to avoid a discontinuity in the energy and the force at the cutoff. These parameters were chosen to prevent crystallization and facilitate trapping

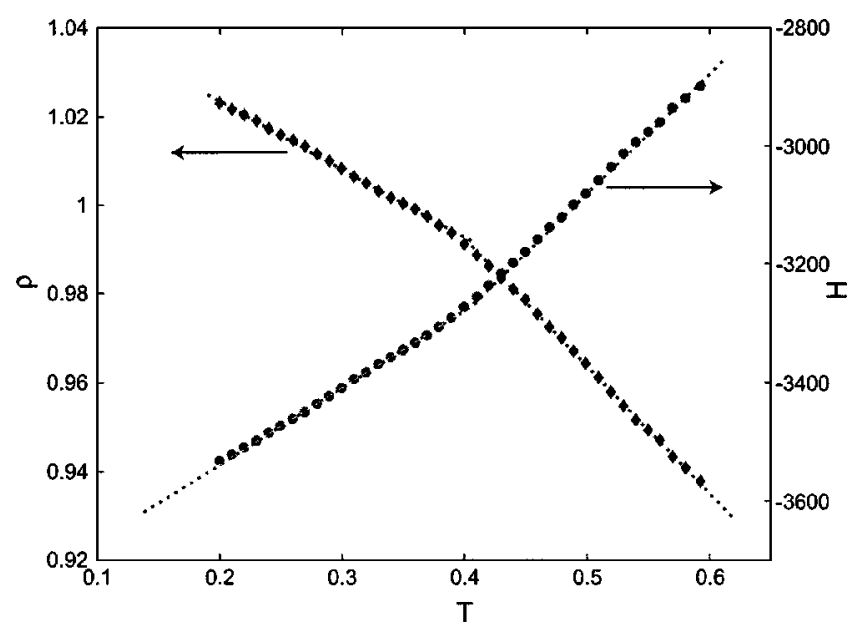

FIG. 1. Specific volume and enthalpy as a function of temperature for the bulk system for a typical simulation.

of the system in an amorphous glassy state. This glassy state is also stable and reproducible under varying geometries such as thin films. ${ }^{34,50}$ The chains used in this work have length $L=32$, which is close to one entanglement length for this model. The number of chains used was 22. All results are reported in reduced variables, i.e., temperature $T^{*}$ $=k T / \varepsilon$ and density $\rho^{*}=N \sigma^{3} / V$.

The equilibration of these systems was carried out using advanced Monte Carlo moves ${ }^{40,41}$ and molecular dynamics in the NPT ensemble at zero pressure. The system was first equilibrated at $T^{*}=1.0$. The temperature was then reduced in steps of 0.1 until $T^{*}=0.6$ and then in steps of 0.05. An apparent glass transition temperature was identified at $T_{g}^{b *}$ $=0.40$ from the change in slope of the specific volume (or energy) versus temperature for a bulk system (Fig. 1). The values of $T_{g}$ determined from both curves agree within the statistical error. The static structure factor of the system, $S(\mathbf{q})$, is shown in Fig. 2 over a range of temperatures from the melt-like state to near the glass transition. From Fig. 2, one observes no qualitative change as $T_{g}$ is approached, as expected for a glass-former.

Microcanonical molecular dynamics simulations were

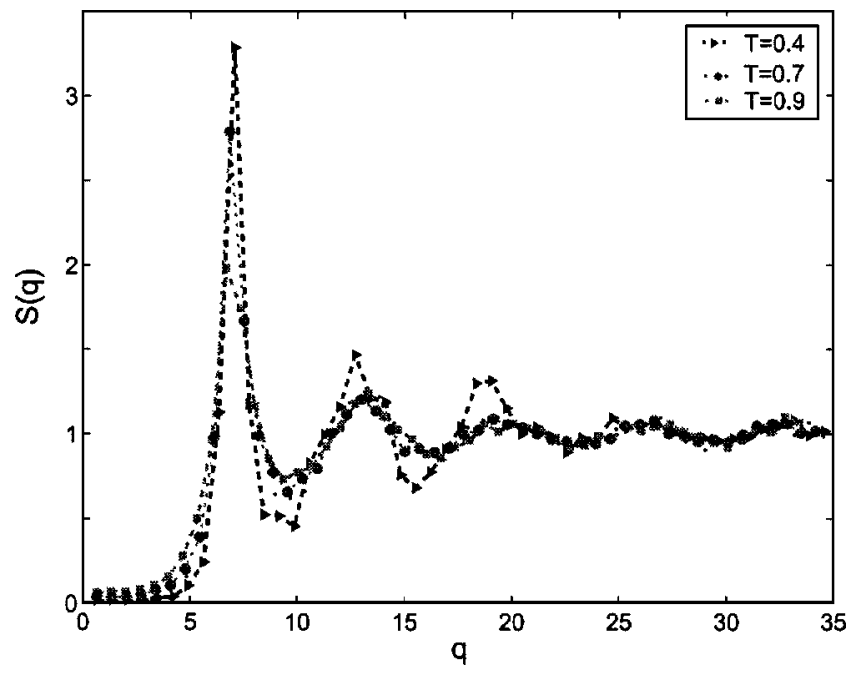

FIG. 2. Static structure factor for the model bulk glass. 


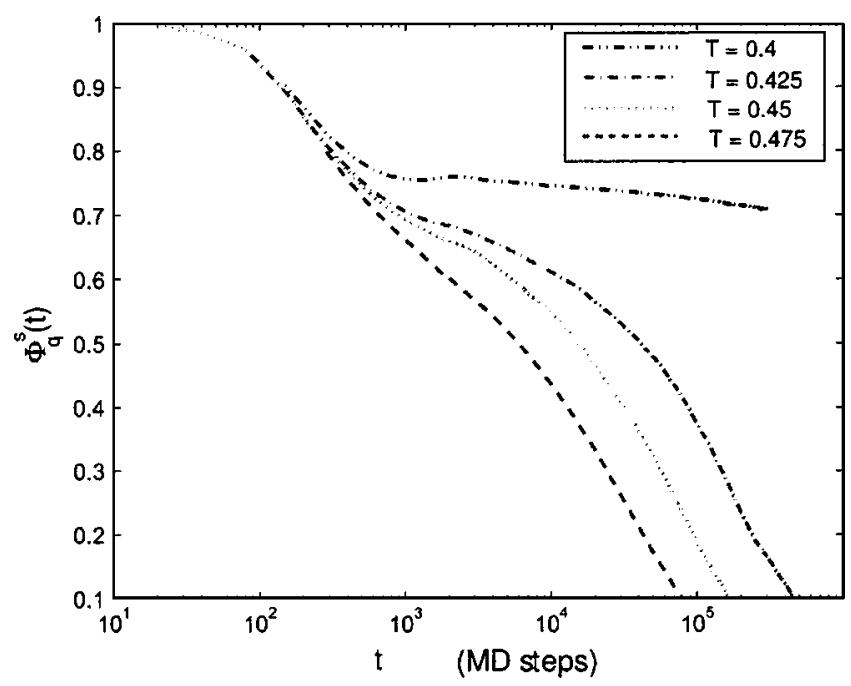

FIG. 3. Incoherent intermediate scattering function for several temperatures approaching $T_{g}$.

then carried out at $T^{*}=0.425$ and at a density of $\rho^{*}=0.984$, with a time step of 0.001 ; configurations were saved every 20 time steps. The ensemble average pressure is zero under these conditions. The choice of this temperature was dictated by several preliminary studies conducted at temperatures ranging from $T^{*}=0.4$ to 0.475 . The incoherent intermediate scattering function $\Phi_{q}^{s}(t)$ is shown in Fig. 3 for several temperatures in this temperature range. This function was calculated at a value of $\mathbf{q}$ corresponding to the first peak in $S(\mathbf{q})$. One can clearly observe the onset of a two-step relaxation with a stretched exponential decay as $T_{g}$ is approached. At temperatures lower than 0.425 , it was found that the time scale for $\alpha$ relaxation $\tau_{\alpha}$, as defined by $\Phi_{q}^{s}\left(\tau_{\alpha}\right)=0.3$, was extremely large, thereby rendering detailed calculations computationally unfeasible for the system sizes considered here. At higher temperatures, the motion of the particles is influenced to a lesser degree by the PEL; $T^{*}=0.425$ was therefore chosen as the temperature for further study. In order to study the PEL of the model glass, it is necessary to minimize the potential energy of the system to the local minima. Such a mapping was proposed by Goldstein ${ }^{29}$ and Stillinger ${ }^{28}$ and has been extensively used to investigate both model glasses $^{30,31}$ as well as atomistic systems. ${ }^{38,42}$ Several algorithms ${ }^{43}$ exist for finding the minimum (or conversely, the maximum) of a function. We employed a conjugate gradient algorithm ${ }^{43}$ for this study. The energy minimization is assumed to have converged if the decrease in energy between subsequent steps is below $10^{-9} \varepsilon$. The final forces on the particles are of the order of $10^{-4} \varepsilon / \sigma$.

\section{AVERAGE LOCAL STRUCTURE OF THE SYSTEM}

This section describes the local structure of the inherent structures of the system in terms of the Voronoi polyhedra and the Delaunay simplices. By construction, a Voronoi polyhedron of a site is the region of space that is nearest to that site than to any other site. The Voronoi polyhedron serves as a measure of the local empty space around a site. These polyhedra possess the property that they completely tile space, i.e., they fill up space without any overlaps or

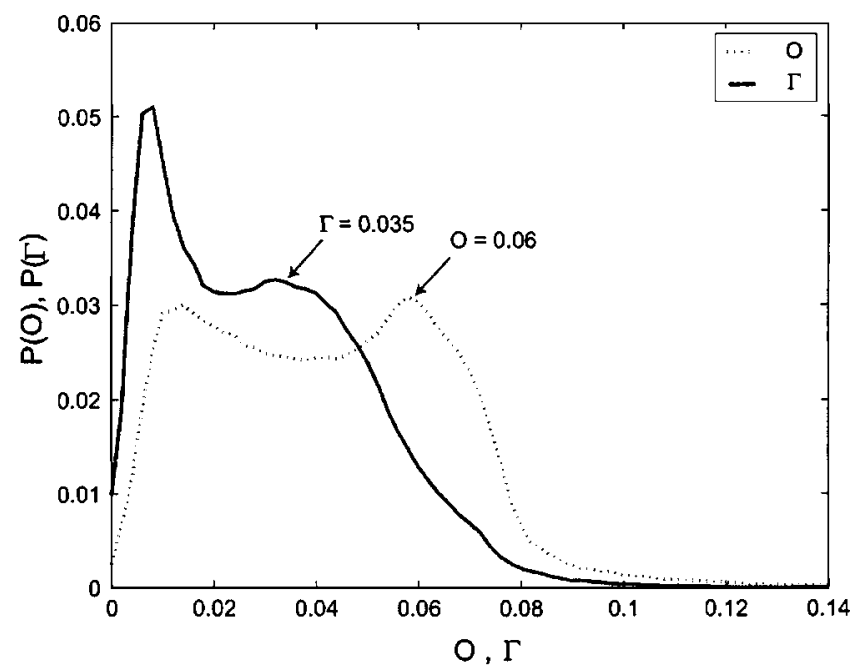

FIG. 4. Distribution of $\Gamma$ and $O$ for the Delaunay simplices.

voids. For a completely amorphous system, every face of a Voronoi polyhedron is shared by two sites, every edge is common to three sites and every vertex of a Voronoi polyhedron is shared by exactly four sites. The tetrahedron formed by these four sites is called a Delaunay simplex (or Delaunay tetrahedron). The Delaunay simplices are the duals of the Voronoi polyhedra, and also possess the property that they tile space. While the Voronoi polyhedra enclose the volume around a particle, the Delaunay simplices enclose the volume between particles. The shape of the Voronoi polyhedra and the arrangement of the Delaunay tetrahedra can provide a measure of the local packing in amorphous systems. Several measures have been used for Voronoi polyhedra; these include the volume $\left(V_{V}\right)$, surface area $\left(A_{V}\right)$, asphericity $\left(S_{V}\right){ }^{17}$ and curvature $\left(C_{V}\right){ }^{15}$ The asphericity and curvature are defined by

$$
\begin{gathered}
S_{V}=\frac{1}{36 \Pi} \frac{A_{V}^{3}}{V_{V}^{2}}-1, \\
C_{V}=\frac{A_{V} \sum_{i} l_{i} \theta_{i}}{24 \pi V_{V}},
\end{gathered}
$$

where $l_{i}$ is the length of edge $i$ of the polyhedron and $\theta_{i}$ is the angle between the normals of the intersecting faces. The value of $S_{V}$ is zero for a sphere and increases with increasing deviation from a spherical shape. For the Delaunay simplex, the measures include the tetrahedricity $(\Gamma),{ }^{17,44}$ octahedricity $(O),{ }^{17}$ and void size $\left(v_{T}\right),{ }^{39,45}$ i.e., the largest void that can be inscribed inside the tetrahedron without overlap with the particles. The units for $l_{i}, A_{V}, V_{V}$, and $v_{T}$ are in terms of $\sigma, \sigma^{2}$, and $\sigma^{3}$, respectively.

Figure 4 shows the distribution of tetrahedricity $\Gamma$ and octahedricity $O$, for the Delaunay simplices obtained from the inherent structures at $T^{*}=0.425$. One can observe a distinct bimodal structure that arises from the presence of both slightly distorted tetrahedra and quart-octahedra (i.e., the tetrahedron formed from one quarter of an octahedron). The tetrahedricity of an ideal quart-octahedron is $\Gamma_{O}=0.05$, and the octahedricity of an ideal tetrahedron is $O_{T}=0.08$. Though 


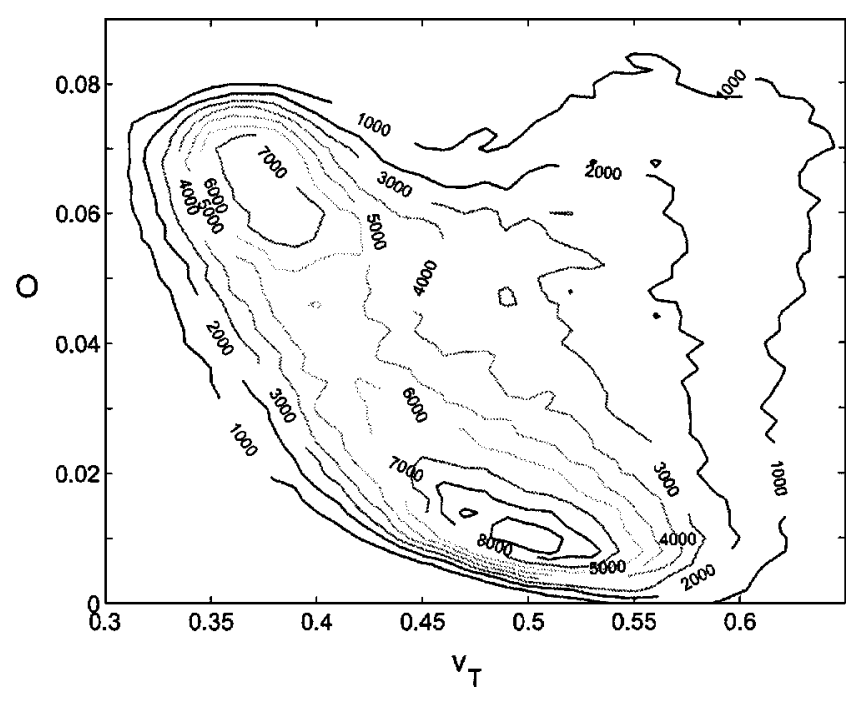

FIG. 5. Distribution of $v_{T}$ vs $O$. The numbers on the contour plot are proportional to the probability, $P\left(v_{T}, O\right)$. The units for $v_{T}$ are in terms of $\sigma^{3}$.

not in exact agreement, the values at the second peaks of the distributions are close to the expected values, and confirm that the second peak in $\Gamma$ arises from quart-octahedra and the second peak in $O$ arises from distorted tetrahedra. Such bimodal distributions have also been observed in model softsphere glasses. ${ }^{39,45}$ Figure 5 shows the two-dimensional distribution of Delaunay simplex void size and the octahedricity. This figure corroborates the previous conclusions, and confirms the existence of two distinct populations of simplices. As expected, the octahedral voids have a larger value of $v_{T}$ than the tetrahedra.

While the Delaunay simplices serve as a useful measure of the local arrangement of particles, an analysis of the local structure in terms of the Voronoi polyhedra reveals greater information about the arrangement of particles around the particle of interest. Figure 6 shows the distribution of the Voronoi volume, surface area, curvature and face areas $A_{F}$, of the polyhedra. One can observe that the distributions of the first three quantities are unimodal and exhibit no specific features. However, for $A_{F}$, one observes a strong peak close to zero, which implies the presence of geometrical neighbors that have a perturbative origin, i.e., arise from distant neighbors ${ }^{44}$ that will cease to be neighbors if the position of the particles is slightly perturbed. Such perturbative artifacts often mask the underlying nature of the local arrangement. We have therefore eliminated such effects by the following procedure:

(1) Construct the complete Voronoi polyhedron for the particle.

(2) Calculate the face areas and the corresponding neighboring particles.

(3) Reconstruct the Voronoi polyhedra by eliminating those particles which lead to face areas below a certain cutoff, $A_{c}$.

We term the new neighbors obtained in this fashion as true geometric neighbors. A similar procedure has also been employed previously to study local arrangements. ${ }^{44}$ Figure 7 shows a dramatic change in the probability distribution of the number of faces as the perturbative faces are eliminated. The distribution does not change further (within the uncertainty) as the cutoff for the elimination is changed from 0.1 to 0.15 . Specifically, after the elimination procedure, we observe the presence of a large fraction of polyhedra with 12 faces, which is a signature of icosahedral ordering. Further, upon closer inspection of the number of edges per face (Fig. 8), we find a large fraction of pentagonal faces, which provides additional evidence for the presence of an underlying icosahedral order in the system. Section IV details the calculation of the metabasins of vibration and investigates the correlation between structural and dynamical aspects.

\section{METABASIN CALCULATION AND INFLUENCE OF STRUCTURE ON DYNAMICS}

In a departure from previous detailed studies of the PEL of model glasses, this work considers systems that are rela-
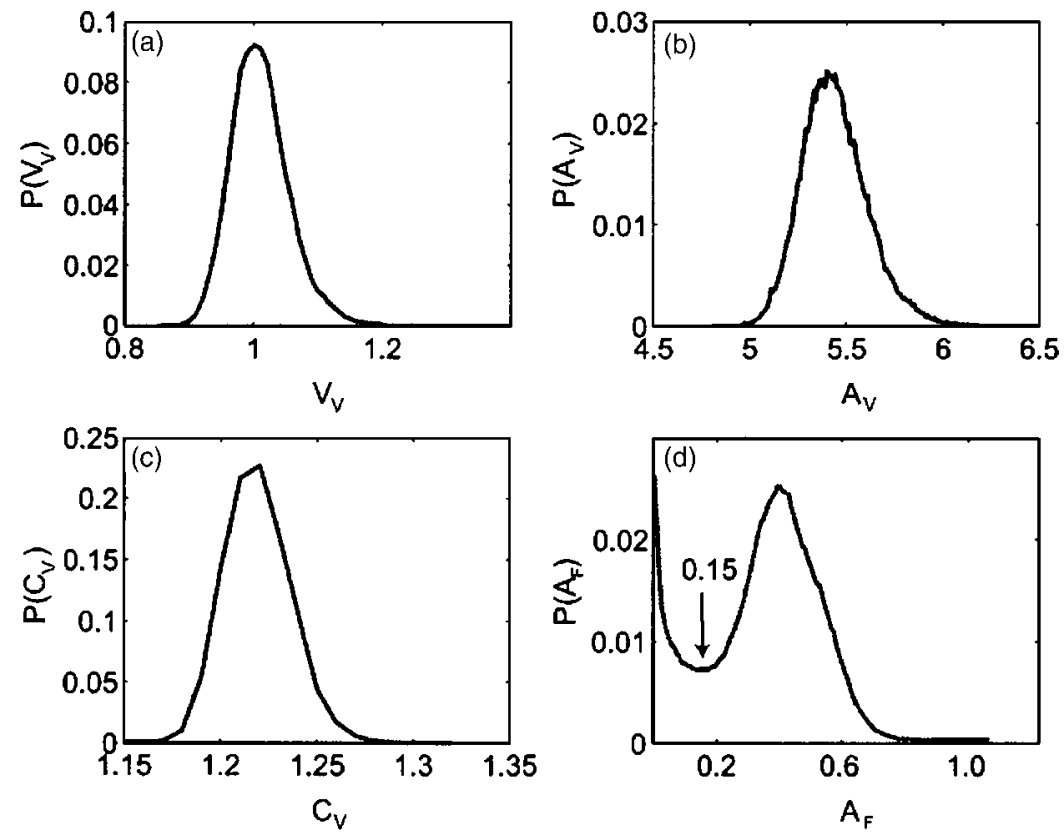

FIG. 6. Distribution of (a) Voronoi volume, (b) surface area of Voronoi polyhedra, (c) curvature of Voronoi polyhedra, and (d) face areas of Voronoi polyhedra. The arrow indicates the face area cutoff $A_{c}=0.15$ below which two sites sharing that face are not considered true neighbors. The units for volume and area are in terms of $\sigma^{3}$ and $\sigma^{2}$, respectively. 


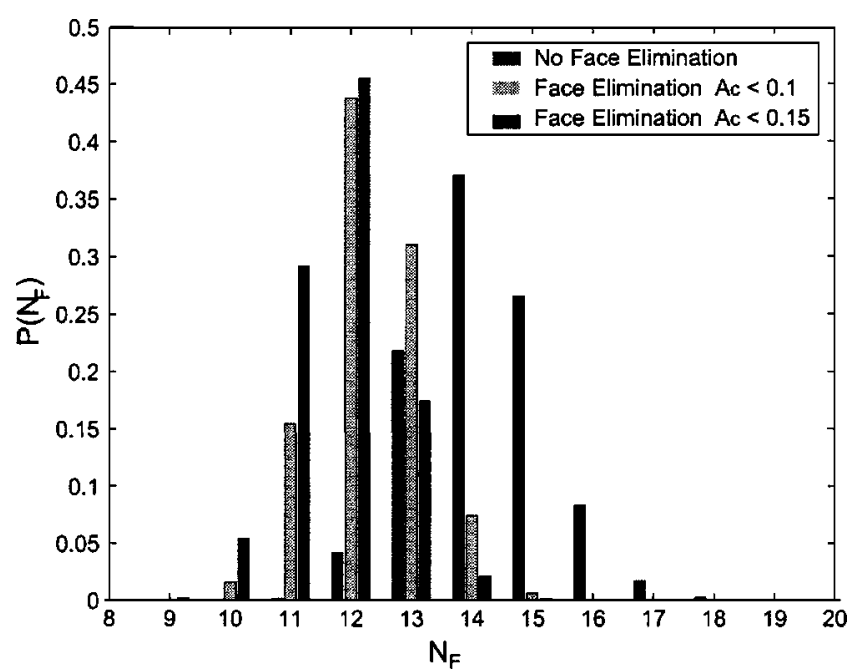

FIG. 7. Probability distribution for number of faces of the Voronoi polyhedra with and without small face elimination.

tively large. Large systems are necessary to reduce finite-size effects when studying a polymeric system. The simulation box length is approximately 1.5 times the average end-to-end distance of the polymer chains. Previous reports have indicated that a large supercooled-liquid system can be decomposed into smaller, weakly interacting subsystems. ${ }^{46}$ Furthermore, the number of minima on the PEL grow exponentially with system size. In such a scenario, relaxations from the different subsystems will superimpose, and it is not possible to identify metabasins solely on the basis of the energies and configurations of the inherent structures, as has been done before. ${ }^{30}$ We have therefore adopted an alternative approach to identify the metabasins for our system. More specifically, a variant of the algorithm proposed by Heuer et al. ${ }^{30}$ is used to track the positions and neighborhood of individual particles, and to identify metabasins of vibration for each particle instead of the entire system. We define that a change in the environment of a particle has occurred if the number or identities of the geometrical neighbors of the particle have changed. Thus at time $t$, a particle $i$ 's state is characterized by

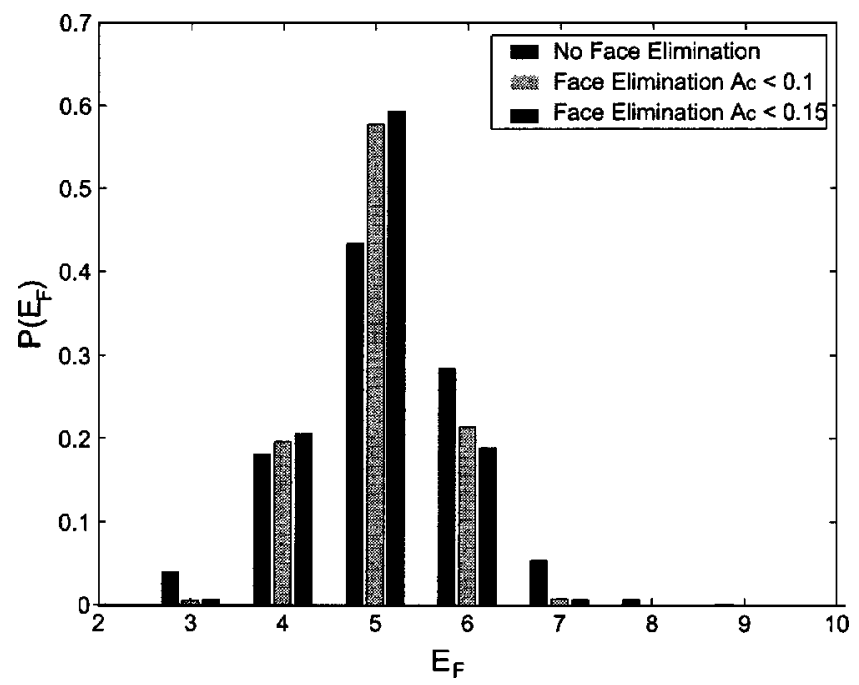

FIG. 8. Probability distribution for number of edges per face of the Voronoi polyhedra with and without small face elimination. the vector $S_{i}(t)=\left[\mathbf{r}_{i}(t), I_{1 t}, \ldots, I_{N_{V}^{i} t}\right]$, where $\mathbf{r}_{i}$ is the position vector of the particle, $I_{j t}$ denotes the Voronoi neighbor $j$ of the particle, and $N_{V}^{i}$ denotes the total number of Voronoi neighbors at time $t$. The algorithm begins by scanning the vector $S_{i}$ over the length of the simulation. Two state vectors are considered different from each other if the displacement between their positions is greater than a certain tolerance, $\delta_{\text {disp }}$, or if there is a change in the list of Voronoi neighbors. During the scan, the times at which each state $k$ occurs are found and a note is made of the time of first occurrence $t_{k f}$, last occurrence $t_{k l}$, number of occurrences $n_{k}$, and fractional occurrence, $f_{k}=n_{k} /\left(t_{k l}-t_{k f}\right)$. Since a metabasin consists of a particle residing in several neighboring local energy minima, the time intervals $\left[t_{k f}, t_{k l}\right]$ overlap with several other time intervals. We follow a variant of the procedure proposed by Heuer et al. $^{30}$ in order to combine these intervals to obtain time periods corresponding to metabasins. Briefly, the procedure is as follows:

(1) Time intervals with a fractional occurrence of less than $1 \%$ are eliminated.

(2) Next, we eliminate the intervals $\left[t_{k f}, t_{k l}\right]$ that are completely contained in another larger time interval.

(3) Two intervals that overlap are combined, either if the length of overlap is greater than half the maximum length of the two intervals, or if the length of overlap is greater than $80 \%$ of the smaller interval.

(4) Two overlapping intervals that do not satisfy the above criterion are split into smaller nonoverlapping intervals as prescribed by Heuer et al. ${ }^{30}$

In some rare cases, we find that certain long intervals consist of small fractional visits, which indicates that the same state vector, $S_{i}$, is revisited after a long gap in time. Further, we reiterate that we only look at particle positions and identity of the neighbors in defining the state point. Thus the same $S_{i}$ could result from a different arrangement of neighbors or from a short-lived fluctuation in the position. We use $f_{k}$ as a simple indicator of such an event and eliminate those intervals, in order to prevent unphysically large metabasins. The next two steps eliminate the short-lived vibrations between local minima within a larger metabasin and combine time intervals of significant overlap into a single metabasin. Figure 9 shows the trajectory of energies of inherent structures generated during the NVE simulation. It is clear that the system does not exhibit the distinct presence of metabasins as defined in earlier studies. ${ }^{47,48}$ However, Fig. 9 shows the typical trajectory of a particle over the same inherent structures. In Fig. 9 one observes that, over several long periods of time, a particle vibrates about a mean position, and infrequently jumps into a different metabasin. The dotted lines show the metabasins identified by our algorithm for a typical trajectory. The length of the dotted line is the size of the metabasin in units of time. In order to ascertain whether the PEL actually influences the dynamics, i.e., if a separation of time scales exists between vibrations and activated jumps, we have examined the correlation of the magnitude of the jumps between successive metabasins in the IS trajectory and the actual molecular dynamics (MD) trajectory. This was 

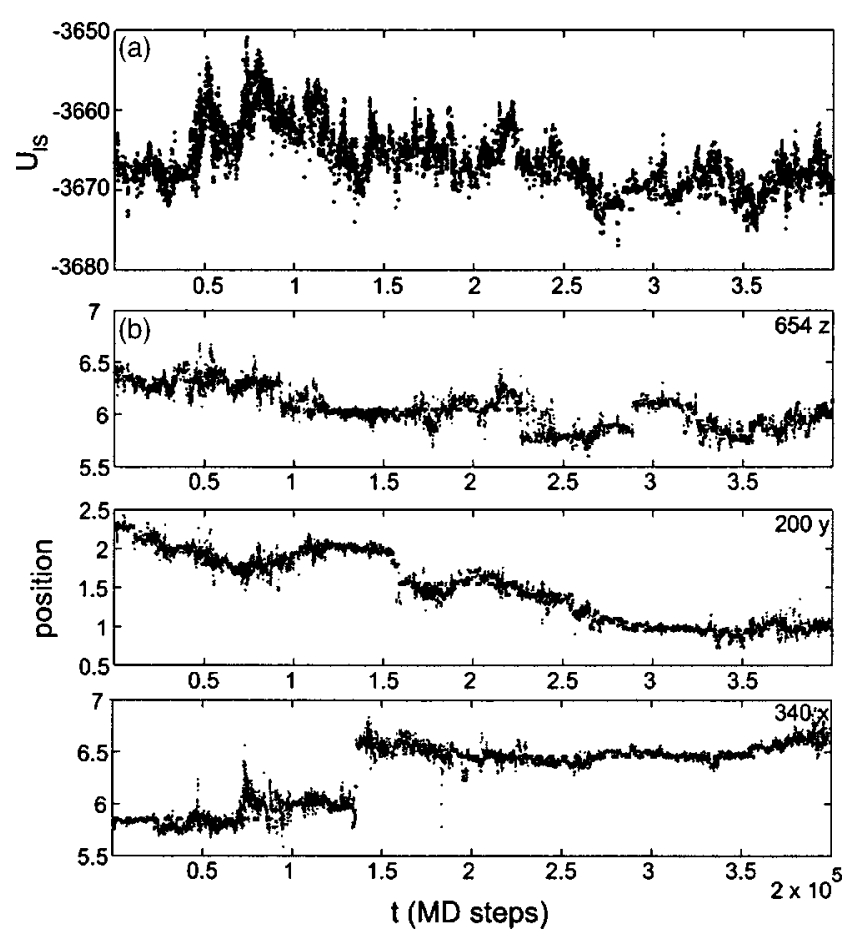

FIG. 9. (a) Trajectory of IS energies, $U_{\text {IS. }}$ (b) Typical trajectories of particle coordinates. The particle numbers and directions are indicated in the topright corner. The dotted lines denote the metabasins calculated for this trajectory.

done as follows: over the time period of the metabasin, the average position of the particle was calculated over the IS trajectory and the MD trajectory. Next, the displacements between the average positions were calculated for successive metabasins, for both trajectories. Figure 10 shows the correlation between the displacements for the two trajectories. We find that the two are in excellent agreement, thereby confirming that the true dynamics are a reflection of the underlying motion on the PEL.

Figure 11 shows the distributions of Voronoi volumes versus the number of neighbors, averaged over the vibrations in a metabasin. As stated earlier, the elimination of perturba-

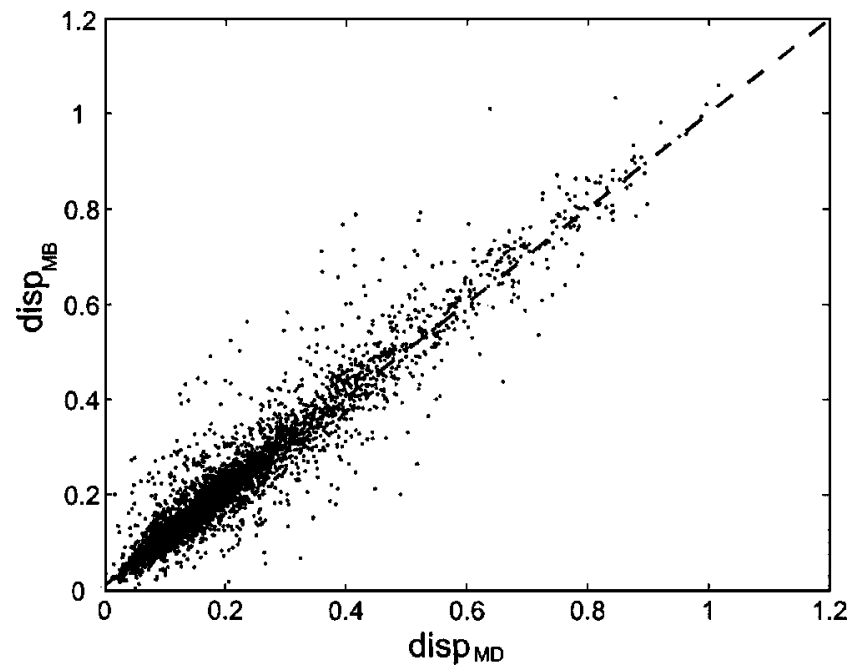

FIG. 10. Correlation of particle displacements between metabasins in the MD trajectory and the IS trajectory.
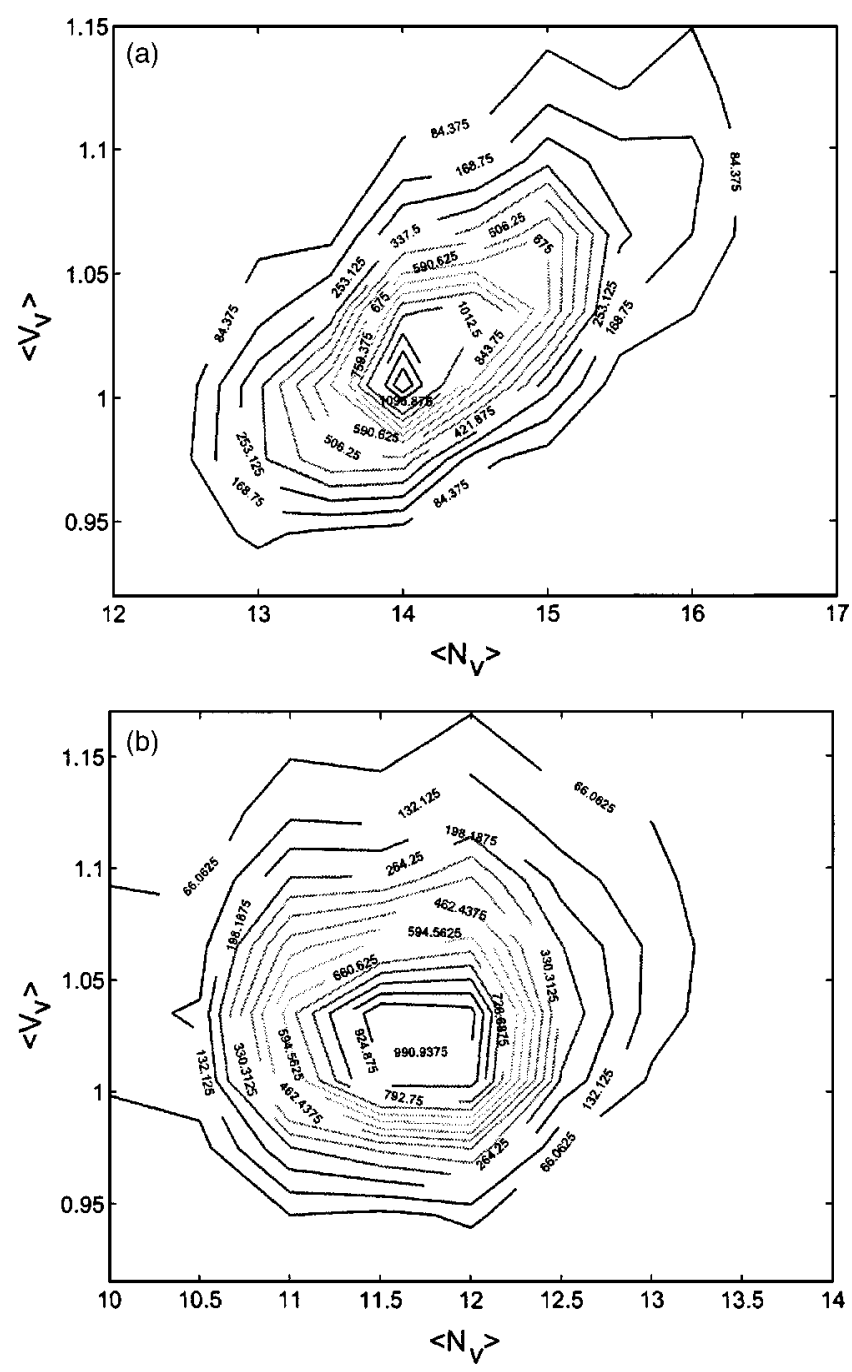

FIG. 11. Distribution of Voronoi volume as a function of number of neighbors, during vibration in a metabasin: (a) without face elimination, and (b) with face elimination with $A_{c}=0.15$. The numbers are proportional to probability $P\left(N_{V}, V_{V}\right)$. The units for volume are in terms of $\sigma^{3}$.

tive faces reveals the presence of a large fraction of 12 coordinated particles. Figure 12 shows the average length of the particle metabasins in units of number of time steps, $\left\langle L_{\mathrm{MB}}\right\rangle$, as a function of the average Voronoi volume of the particle and number of neighbors during its residence in the metabasin. One can observe a clear correlation, with shorter metabasins associated with larger $\left\langle v_{V}\right\rangle$. The value of the average Voronoi volume over all the sites in the system (i.e., number density) is 1.02 . Note that in the construction of this figure, the small face elimination was not performed. If such an elimination is conducted, then the distribution shifts toward 12 neighbors, as shown in Fig. 7. Such a Voronoi cell is reminiscent of icosahedral ordering, and the results indicate that a greater degree of icosahedral ordering leads to a longevity of the particle metabasins. Figure 13 shows the distribution of average tetrahedricity in a metabasin, $\langle\Gamma\rangle$, for sites belonging to metabasins with different $L$. The distributions shift to the left as the size of the metabasins increase, revealing a distinct influence of $\Gamma$ on metabasin size. 


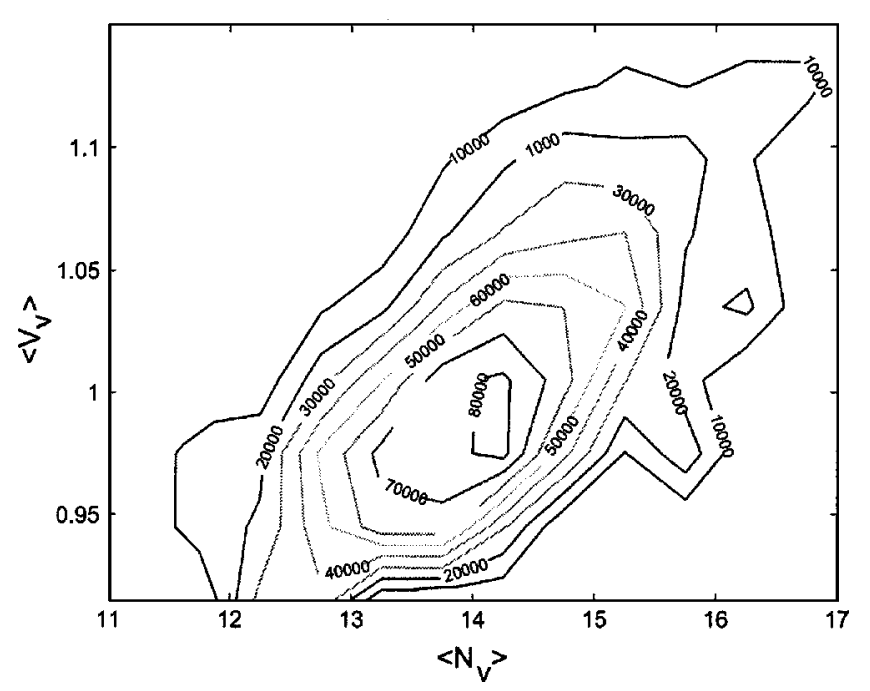

FIG. 12. Average length $\left\langle L_{\mathrm{MB}}\right\rangle$ of a metabasin as a function of Voronoi volume and number of geometrical neighbors. The units in the contour plot are in terms of number of MD time steps.

\section{INFLUENCE OF COOPERATIVITY ON STRUCTURE}

It is well established that the dynamics in supercooled liquids exhibit a cooperative nature, ${ }^{8,35,49}$ even at the level of single transitions between neighboring inherent structures. ${ }^{34}$ While the results presented so far demonstrate that a correlation exists between dynamics and structure based on single particle properties, a study of the structure of cooperatively rearranging regions could provide even greater insights into the microscopic origins of heterogeneous dynamics. Our work suggests that some insights into the nature of collective motion on the structure of the system could be obtained by studying the vibrations at the IS in the harmonic limit, i.e., the normal modes of vibration, and their influence on the tetrahedricity of the system, $\Gamma_{s}$. The tetrahedricity of the inherent structures can be expanded in a second-order Taylor's series:

$$
\Gamma_{s}=\Gamma_{s 0}-\mathbf{F}_{\Gamma} \cdot \boldsymbol{\Delta}+\frac{1}{2} \boldsymbol{\Delta}^{T} \cdot \mathbf{H}_{\Gamma} \cdot \boldsymbol{\Delta},
$$

where $\Gamma_{s 0}$ is the tetrahedricity of the system at the minimum, $\mathbf{F}_{\Gamma}$ and $\mathbf{H}_{\Gamma}$ denote the force and Hessian equivalents of the

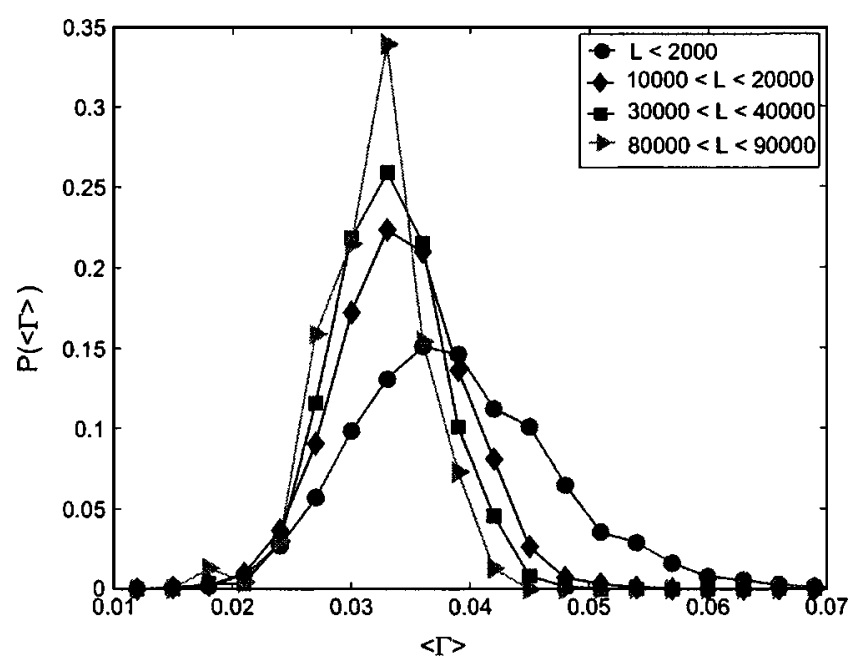

FIG. 13. Distribution of average tetrahedricity for particles vibrating in metabasins of different lengths. The units in the legend are in terms of number of MD time steps. tetrahedricity, and $\boldsymbol{\Delta}$ is the displacement field. The expressions for $\mathbf{F}_{\Gamma}$ and $\mathbf{H}_{\Gamma}$ are given in the following:

$$
\begin{aligned}
& F_{m \alpha}=\frac{12}{15} \sum_{t} \sum_{p}\left(\frac{6}{\bar{l}_{t}^{2}}-\frac{\overline{l_{t}^{2}}}{\bar{l}_{t}^{3} r_{m p}}\right) r_{m p \alpha}\left(1-\delta_{m p}\right), \\
& \frac{\partial^{2} \Gamma_{s}}{\partial r_{\alpha}^{m} \partial r_{\beta}^{n}}=\frac{12}{15} \sum_{t} \sum_{p}\left[\frac{1}{\bar{l}_{t}^{2}}-\frac{\overline{l_{t}^{2}}}{\overline{l_{t}^{3}}\left|r_{m p}\right|}\right]\left(\delta_{m n}-\delta_{p n}\right) \\
& \times \delta_{\alpha \beta}\left(1-\delta_{m p}\right)+\frac{12}{15} \sum_{t} \sum_{p} \frac{r_{m p \alpha} r_{m p \beta}}{\left|r_{m p}\right|^{3}}\left(\delta_{m n}-\delta_{p n}\right) \\
& \times \delta_{\alpha \beta}\left(1-\delta_{m p}\right)-\sum_{t} \frac{4}{15 \bar{l}_{t}^{3}} \sum_{p} r_{m p \alpha}\left(1-\delta_{m p}\right) \\
& \times \sum_{k} \frac{r_{n k \beta}}{\left|r_{n k}\right|}\left(1-\delta_{n k}\right)-\sum_{t} \frac{4}{15 \bar{l}_{t}^{3}} \sum_{p} r_{m p \alpha}\left(1-\delta_{m p}\right) \\
& \times \sum_{p} \frac{r_{m p \alpha}}{\left|r_{m p}\right|}\left(1-\delta_{m p}\right) \sum_{k} r_{n k \beta}\left(1-\delta_{n k}\right) \\
& +\sum_{t} \frac{6 \overline{l_{t}^{2}}}{15 \bar{l}_{t}^{3}} \sum_{p} \frac{r_{m p \alpha}}{\left|r_{m p}\right|}\left(1-\delta_{m p}\right) \sum_{k} \frac{r_{n k \beta}}{\left|r_{n k}\right|}\left(1-\delta_{n k}\right),
\end{aligned}
$$

where $t$ is the index over the tetrahedra containing particle $m, p$ and $k$ are the indices of the particles in tetrahedron $t, \bar{l}_{t}$ is the average length of the edges in tetrahedron $t$, and $\alpha, \beta$ denote the Cartesian directions. In a previous publication ${ }^{50}$ we discussed the cooperative nature of the modes and the significance of the Boson peak on the observed dynamics in glasses. In simulations, one can obtain the Boson peak by calculating the dynamic structure factor $S_{\text {inc }}(\mathbf{q}, \omega)$, or by diagonalizing the Hessian matrix at the IS. Figure 14 shows the results obtained from both methods; they are in excellent agreement, with a clear Boson peak present at $\omega \approx 2.5$. The advantage of the Hessian method is that one can obtain the eigenvectors to examine the nature of the motion with a particular frequency. Each eigenvector $\mathbf{Q}_{k}$ has length $3 N$, which can be thought of as $N$ displacement vectors $\mathbf{q}_{k}^{\mathbf{i}}$ acting on the particles in the system:

$$
\mathbf{Q}_{k}=\left[\mathbf{q}_{k}^{1}, \ldots, \mathbf{q}_{k}^{\mathrm{N}}\right] .
$$

These particle vectors give the direction and displacement of the particles in a particular eigenmode. The eigenvector describes the collective instantaneous vibrational dynamics of the system, since it gives the direction of displacement of all the particles in the system. One can investigate the nature of the vibrations on the local structure by studying how $\Gamma$ varies as the system is moved along a normal mode of frequency $\omega$. This can be quantified by

$$
\begin{aligned}
\Delta \Gamma_{s}(\omega)= & \Gamma_{s}(\omega)-\Gamma_{s 0}=-\mathbf{F}_{\Gamma} \cdot \mathbf{Q}(\omega) \\
& +\frac{1}{2} \mathbf{Q}(\omega)^{T} \cdot \mathbf{H}_{\Gamma} \cdot \mathbf{Q}(\omega)=\Delta \mathbf{F}_{\Gamma}(\omega)+\Delta \mathbf{H}_{\Gamma}(\omega),
\end{aligned}
$$

where the frequency-dependent contributions due to the force and the Hessian are denoted as $\Delta \mathbf{F}_{\Gamma}(\omega)$ and $\Delta \mathbf{H}_{\Gamma}(\omega)$, respectively. Figure 15 shows these contributions for the low 

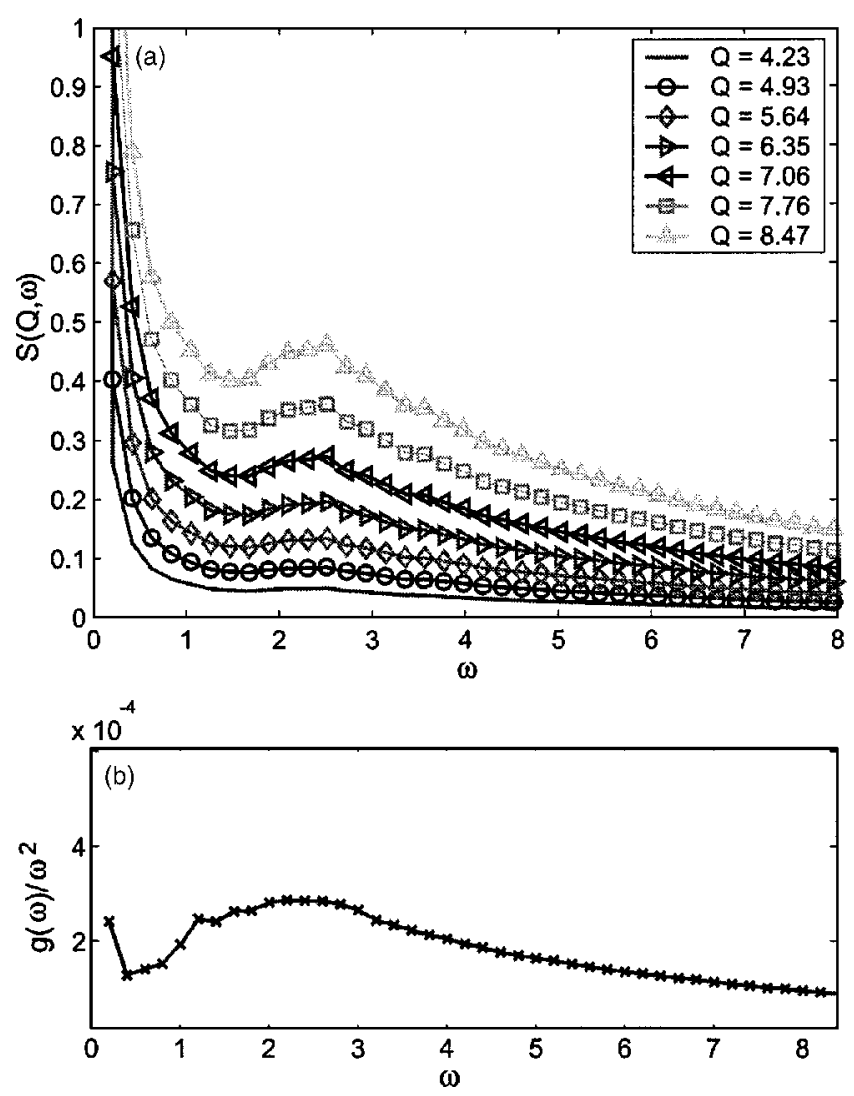

FIG. 14. Calculating the location of the Boson peak from (a) dynamic structure factor and (b) Hessian diagonalization. The units for $\mathbf{q}$ are in terms of $\sigma^{-1}$.

frequency modes. One can make the observation that the low frequency modes (directions with lowest curvature of potential energy on the PEL) correspond to the greatest change in local structure measured in terms of the tetrahedricity. However, a distinct change in the nature of the curve is observed at $\omega \approx 2$, which is close to the Boson peak frequency for this model. Similar observations were also made by Schober et $a l .{ }^{17}$ for soft-sphere glasses. Thus, we find that similar measures of local structure (tetrahedricity in this case) influence the motion in both the vibrational limit as well as in the limit governing the transitions between metabasins.

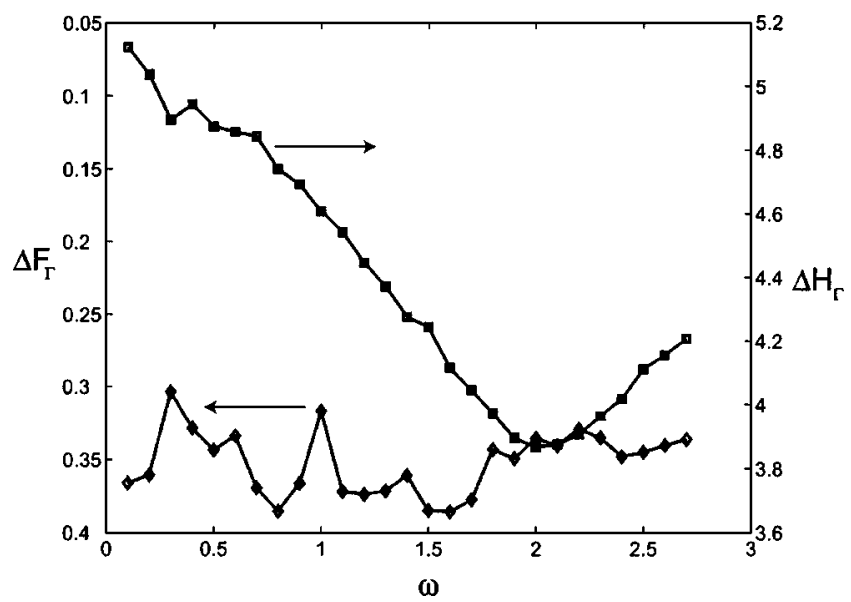

FIG. 15. Change of system tetrahedricity as a function of normal mode frequency.

\section{DISCUSSION AND CONCLUSIONS}

The model glass forming polymer considered in this work exhibits the canonical features expected from a glassformer, such as a well-defined apparent glass transition temperature and a two-step nonexponential relaxation behavior over time. For each particle, metabasins of vibration have been identified in the inherent structure trajectory using an algorithm based on the position and neighborhood of the particles. We have investigated the relationship between the local structure around a monomer for this glass former and its period of vibration on the PEL. We find that its Voronoi volume and coordination number correlate with its residence time in the metabasin. Our results provide concrete evidence that activated dynamics are governed by both the local free volume and the geometry of the free volume. Recent results by Cooper et al. have shown that the starting configuration influences the spatial distribution of dynamic heterogeneities. ${ }^{9}$ However, the specific aspect of the local structure influencing the dynamics was not investigated. Our finding that specific structural arrangements have a direct impact on the observed dynamics make this study novel. Since certain local environments can lead to greater tendency for motion, our results also aid in understanding the origin of "mobile excitations" in the dynamically facilitated models for glasses. ${ }^{12,13}$ It has been previously hypothesized that frustration of nonspace filling structures ${ }^{51,52}$ could be at the origin of dynamic heterogeneities, with motion occurring along the domain walls between preferred local structures. Domain frustration has also formed the basis of a recent thermodynamic theory of glass transition. ${ }^{53}$ Interestingly, an increase in the coordination number has been shown to influence the dynamics in supercooled water. ${ }^{38}$ These observations were rationalized through geometric frustration arguments in a tetrahedral network. Atomistic simulations of a $m$-toluidine have revealed the presence of pairs of strongly $\mathrm{H}$-bonded molecules that exhibit slower dynamics than the rest of the system. ${ }^{37}$ However, both of these systems exhibit strong directional interactions in the form of $\mathrm{H}$ bonding, and it is not clear from the above studies whether such conclusions extend to glassy systems in general, including polymeric glasses. Studies of a model potential ${ }^{36}$ that imitates the structure of metallic glass formers have also shown the influence of local structure on the dynamics, with substantial reduction in the mean-square displacement for particles present in an icosahedral ordering. However, the potential is constructed to favor icosahedral ordering ${ }^{54}$ in the first coordination shell. Our system lacks specific directional interactions, and our results indicate that the specific preferred structural arrangements have a clear and well-defined influence on glassy dynamics in general. Identification of such structural motifs in different glass formers could considerably improve our understanding of the influence of chemical structure on the dynamics in glass formers.

Furthermore, we have also found that the vibrations at an inherent structure (not a metabasin) exhibit a nontrivial dependence on the structure, which changes at a frequency close to the Boson peak for this model. In a previous study ${ }^{50}$ using the same model, we demonstrated the cooperative 
character of the vibrations of the low frequency modes. From earlier studies on soft-sphere glasses ${ }^{17,55}$ it is known that the low frequency modes are concentrated on $O(10)$ sites that are spatially correlated and exhibit structural differences from the average structure of the glass. ${ }^{56}$ Excitation of the low frequency modes in $\mathrm{Ni}_{81} \mathrm{P}_{19}$ glass ${ }^{57}$ was also found to result in well-defined atomic motions with cooperative character. Other studies have shown that short time vibrational dynamics correlate both with the local free volume ${ }^{58}$ and the long-time relaxation of several small-molecule and polymeric glass formers. ${ }^{59-61}$ The intensity at the Boson peak frequency has also been interpreted in terms of the length scale of the frozen-in fluctuations of the local elastic constants. ${ }^{62}$ Our results indicate that motions at both short and long time scales exhibit a dependence on the tetrahedricity which is a measure of the local structure and distortion. We believe that our finding of a common structural origin provides a mechanistic explanation for the experimental observations $^{59-61}$ that relate the nature of the short-time vibrational dynamics to the $\alpha$ relaxation.

\section{ACKNOWLEDGMENTS}

Financial support from the US Department of Energy (Contract No. DE-FG02-9914961) is gratefully acknowledged. The authors would also like to thank Professor Mark Ediger for several interesting discussions during the course of this work.

${ }^{1}$ M. H. Cohen and G. S. Grest, Phys. Rev. B 20, 1077 (1979).

${ }^{2}$ T. G. Fox and P. J. Flory, J. Appl. Phys. 21, 581 (1950).

${ }^{3}$ A. K. Doolittle, J. Appl. Phys. 22, 1471 (1951).

${ }^{4}$ R. L. Leheny et al., J. Chem. Phys. 105, 7783 (1996).

${ }^{5}$ F. Varnik, J. Baschnagel, and K. Binder, Eur. Phys. J. E 8, 195 (2002).

${ }^{6}$ M. Ediger, Annu. Rev. Phys. Chem. 51, 99 (2000).

${ }^{7}$ H. Sillescu, J. Non-Cryst. Solids 243, 81 (1999).

${ }^{8}$ C. Donati et al., Phys. Rev. Lett. 80, 2338 (1998).

${ }^{9}$ A. Widmer-Cooper, P. Harrowell, and H. Fynewever, Phys. Rev. Lett. 93, 135701 (2004).

${ }^{10}$ S. C. Glotzer, J. Non-Cryst. Solids 274, 342 (2000).

${ }^{11} \mathrm{~S}$. Butler and P. Harrowell, J. Chem. Phys. 95, 4454 (1991).

${ }^{12}$ J. P. Garrahan and D. Chandler, Phys. Rev. Lett. 89, 035704 (2002).

${ }^{13}$ L. Berthier and J. P. Garrahan, Phys. Rev. E 68, 041201 (2003).

${ }^{14}$ N. N. Medvedev, A. Geiger, and W. Brostow, J. Chem. Phys. 93, 8337 (1990).

${ }^{15}$ J. C. G. Montoro and J. L. F. Abascal, J. Phys. Chem. 97, 4211 (1993).

${ }^{16}$ Y. Hiwatari and T. Saito, J. Chem. Phys. 81, 6044 (1984).

${ }^{17}$ V. A. Luchnikov, N. N. Medvedev, Y. I. Naberukin, and H. R. Schober, Phys. Rev. B 62, 3181 (2000).

${ }^{18}$ V. P. Voloshin, Y. I. Naberukhin, and N. N. Medvedev, J. Chem. Phys. 102, 4981 (1995).

${ }^{19}$ Y. Hiwatari, J. Chem. Phys. 76, 5502 (1982).

${ }^{20}$ T. Kondo and K. Tsumuraya, J. Chem. Phys. 94, 8220 (1991).
${ }^{21}$ P. J. Steinhardt, D. R. Nelson, and M. Ronchetti, Phys. Rev. Lett. 47, 1297 (1981).

${ }^{22}$ H. Jonsson and H. C. Andersen, Phys. Rev. Lett. 60, 2295 (1988).

${ }^{23}$ T. Kondo, K. Tsumuraya, and M. S. Watanabe, J. Chem. Phys. 93, 5182 (1990).

${ }^{24}$ K. L. Ngai and C. M. Roland, Macromolecules 26, 6824 (1993).

${ }^{25}$ C. M. Roland and K. L. Ngai, J. Non-Cryst. Solids 172, 868 (1994).

${ }^{26}$ V. P. Privalko et al., J. Chem. Phys. 112, 5254 (2000).

${ }^{27}$ R. Bohmer, K. L. Ngai, C. A. Angell, and D. J. Plazek, J. Chem. Phys. 99, 4201 (1993).

${ }^{28}$ F. H. Stillinger and T. A. Weber, Phys. Rev. A 28, 2408 (1983).

${ }^{29}$ M. Goldstein, J. Chem. Phys. 51, 3728 (1969).

${ }^{30}$ B. Doliwa and A. Heuer, Phys. Rev. E 67, 031506 (2003).

${ }^{31}$ S. Sastry, P. G. Debenedetti, and F. H. Stillinger, Nature (London) 393, 554 (1998).

${ }^{32}$ T. Schrøder, S. Sastry, J. Dyre, and S. Glotzer, J. Chem. Phys. 112, 9834 (2000).

${ }^{33}$ M. Vogel, B. Doliwa, A. Heuer, and S. C. Glotzer, J. Chem. Phys. 120, 4404 (2004).

${ }^{34}$ T. S. Jain and J. J. de Pablo, Phys. Rev. Lett. 92, 155505 (2004).

${ }^{35}$ C. Donati et al., Phys. Rev. E 60, 3107 (1999).

${ }^{36}$ M. Dzugutov, S. I. Simdyankin, and F. H. M. Zetterling, Phys. Rev. Lett. 89, 195701 (2002).

${ }^{37}$ R. Chelli et al., J. Chem. Phys. 116, 6205 (2002).

${ }^{38}$ N. Giovambattista et al., Phys. Rev. E 65, 051402 (2002).

${ }^{39}$ S. Sastry, P. G. Debenedetti, and F. H. Stillinger, Phys. Rev. E 56, 5533 (1997).

${ }^{40}$ F. Escobedo and J. J. de Pablo, J. Chem. Phys. 102, 2636 (1994).

${ }^{41}$ F. Escobedo and Z. Chen, J. Chem. Phys. 113, 11382 (2000).

${ }^{42}$ S. Mossa et al., Phys. Rev. E 65, 041205 (2002).

${ }^{43}$ W. H. Press, S. A. Teukolsky, W. T. Vetterling, and B. P. Flannery, Numerical Recipes in C (Cambridge University Press, New York, 1988).

${ }^{44}$ W. Brostow, M. Chybicki, R. Laskowski, and J. Rybicki, Phys. Rev. B 57, 13448 (1998).

${ }^{45}$ J. L. Finney and J. Wallace, J. Non-Cryst. Solids 43, 165 (1981).

${ }^{46}$ S. Buchner and A. Heuer, Phys. Rev. Lett. 84, 2168 (2000).

${ }^{47}$ A. Heuer and B. Doliwa, Phys. Rev. E 67, 030501 (2003).

${ }^{48}$ R. A. Denny, D. R. Reichman, and J.-P. Bouchaud, Phys. Rev. Lett. 90, 025503 (2003).

${ }^{49}$ Y. Gebremichael, T. Schrøder, F. Starr, and S. Glotzer, Phys. Rev. E 64, 051503 (2003).

${ }^{50}$ T. S. Jain and J. J. de Pablo, J. Chem. Phys. 120, 9371 (2004).

${ }^{51}$ F. H. Stillinger, J. Chem. Phys. 89, 6461 (1988).

${ }^{52}$ C. Frank, Proc. R. Soc. London, Ser. A 215, 43 (1952).

${ }^{53}$ D. Kivelson and G. Tarjus, Philos. Mag. B 77, 245 (1998).

${ }^{54}$ M. Dzugutov, Phys. Rev. A 46, R2984 (1992).

${ }^{55}$ C. Oligschleger and H. R. Schober, Solid State Commun. 93, 1031 (1995).

${ }^{56}$ B. B. Baird and H. R. Schober, Phys. Rev. Lett. 66, 636 (1991).

${ }^{57}$ L. D. Van Ee, B. J. Thijsse, and J. Sietsma, J. Non-Cryst. Solids 205, 641 (1996).

${ }^{58}$ F. W. Starr, S. Sastry, J. F. Douglas, and S. C. Glotzer, Phys. Rev. Lett. 89, 125501 (2002).

${ }^{59}$ K. L. Ngai, A. Sokolov, and W. Steffen, J. Chem. Phys. 107, 5268 (1998).

${ }^{60}$ K. L. Ngai, Philos. Mag. 84, 1341 (2004).

${ }^{61}$ T. Scopigno, G. Ruocco, F. Sette, and G. Monaco, Science 302, 849 (2003).

${ }^{62}$ A. P. Sokolov et al., Phys. Rev. Lett. 78, 2405 (1997). 